\title{
Comparación de dos métodos de criopreservación de semen porcino. Efectos sobre la calidad seminal
}

\author{
Pérez, M.C. ${ }^{1}$; Petrinelli, A. ${ }^{\text {; }}$ Rodríguez, P.C. ; Satorre, M.M. ${ }^{1}$; Breininger, E. ${ }^{1,2}$ \\ ${ }^{1}$ Fac. Cs. Veterinarias, Univ. de Buenos Aires'. Consejo Nac. Investig. \\ Científ.\& Técn. (CONICET)². Chorroarín 280, Buenos Aires, Argentina. \\ E-mail: ebreininger@fvet.uba.ar
}

\begin{abstract}
Resumen
Pérez, M.C.; Petrinelli, A.; Rodríguez, P.C.; Satorre, M.M.; Breininger, E.: Comparación de dos métodos de criopreservación de semen porcino. Efectos sobre la calidad seminal. Rev. Vet. 30: 1, 23-27, 2019. Cerca del 40\% de la carne roja que se consume en el mundo es provista por los cerdos. Los productores porcinos de nuestro país se encuentran ante el enorme desafío de lograr mejoras productivas con un desarrollo económico sustentable y eficiente, así como implementar tecnologías reproductivas, como la inseminación artificial y la criopreservación de semen. Tales adelantos aún presentan enormes problemas en esta especie. El objetivo de este trabajo fue comparar dos métodos simples de congelamiento para semen porcino, evaluando los parámetros de calidad seminal al momento del descongelado. Las muestras de semen fueron criopreservadas en pajuelas siguiendo el protocolo descripto por Peña y colaboradores en 2003, realizando (grupo tratamiento) o no (grupo control) una modificación en el paso final de congelamiento posterior al envasado en las pajuelas. Las evaluaciones de motilidad y vitalidad se realizaron por microscopía óptica en platina termostatizada y por las técnicas de eosina/nigrosina y la combinación del colorante supravital azul tripán con microscopía de contraste interferencial diferencial, respectivamente. La criocapacitación se evaluó por la técnica fluorescente de clorotetraciclina (CTC). Los resultados de los diferentes experimentos fueron analizados por la prueba $t$ de Student. Las muestras del grupo tratamiento evidenciaron una motilidad significativamente más alta $(\mathrm{p}<0,05)$ y una criocapacitación significativamente más baja $(\mathrm{p}<0,05)$ que la del grupo control. El porcentaje de espermatozoides vivos en las muestras del grupo tratamiento $(33 \pm 3)$ resultó el doble que el del grupo control $(16 \pm 2)$. Nuestros resultados demuestran que el método de congelamiento propuesto permite obtener, al descongelado, muestras de una mayor calidad que las obtenidas al descongelar muestras que hayan sido congeladas con el método tradicional.
\end{abstract}

Palabras clave: porcino, semen, criopreservación, calidad seminal.

\begin{abstract}
Pérez, M.C.; Petrinelli, A.; Rodríguez, P.C.; Satorre, M.M.; Breininger, E.: Comparing two simple cryopreservation methods for porcine sperm. Effect on sperm quality parameters. Rev. Vet. 30: 1, 23-27, 2019. About 40\% of red meat consumed around the world is porcine meat. Argentinean producers face the big challenge of achieving productive improvements with efficient and sustainable economic development and reproductive technologies, like artificial insemination and sperm cryopreservation (that still has many problems in this specie), seems to be an essential element for achieving them. The aim of this work was to compare two different simple cryopreservation methods for porcine sperm, evaluating sperm quality parameters at the moment of thawing. Sperm samples were cryopreserved following Peña et al. protocol (2003), performing (treatment group) or not (control group) a modification during the final step of cryopreservation after the straws' packing. Sperm motility and viability of ejaculated and thawed sperm were evaluated by optic microscopy with thermal stage and the combination of the blue trypan supravital technique and differential interferential contrast microscopy, respectively. Cryocapacitation was evaluated by the chlortetracycline technique (CTC). The results of the different experiments were analyzed by t-student test. The samples of the treatment group showed a significantly higher $(\mathrm{p}<0.05)$ sperm motility and a significantly lower $(\mathrm{p}<0.05)$ cryocapacitation than the control group. The percentage of live spermatozoa in samples of the treatment group (33 \pm 3 ) doubled those in the control group (16 \pm 2$)$. Our results demonstrate that the proposed method of cryopreser-
\end{abstract}


vation allows obtaining, at thawing, higher quality sperm samples than the ones obtained with the traditional method of cryopreservation.

Key words: porcine, sperm, cryopreservation, sperm quality.

\section{INTRODUCCIÓN}

La carne de cerdo representa hoy cerca del $40 \%$ de la carne roja que se consume en el mundo, proyectándose a nivel mundial un consumo de $15,3 \mathrm{~kg} /$ habitante/ año para el $2030^{12}$. En los últimos años, en nuestro país se ha incrementado sustancialmente su consumo, llegando en 2015 a unos $11,30 \mathrm{~kg} /$ habitante/año ${ }^{10}$. En tal sentido, los productores porcinos se encuentran ante el enorme desafío de lograr mejoras productivas con un desarrollo económico sustentable y eficiente ${ }^{15}$.

La implementación de tecnologías reproductivas resulta un componente esencial para lograr un buen posicionamiento productivo ${ }^{16}$. La inseminación artificial (IA) es la técnica más importante para el mejoramiento genético de los animales y es una práctica corriente en los principales países productores de cerdos, sustituyendo prácticamente a la monta natural con niveles superiores al 90\% en Europa y en EEUU. En Sudamérica se ha registrado un importante avance en los últimos años, llegando a casi $100 \%$ en Chile y $66 \%$ en Brasil ${ }^{9}$.

En nuestro país las situación es diferente. Si bien los niveles de producción se han incrementado en un $160 \%$ en la última década ${ }^{10}$, el uso de la IA no ha seguido el mismo camino, mostrando aún bajos niveles de utilización. En Argentina, la utilización de esta técnica presenta un problema relacionado con el tiempo de conservación de los espermatozoides, que es de aproximadamente una semana para el semen refrigerado y que llevaría a problemas en el transporte de las dosis seminales debido a lo extenso de nuestro territorio.

Una posible solución a este inconveniente se encuentra en otra biotecnología muy asociada a la IA, que es la criopreservación de semen. A diferencia de lo que ocurre en otras especies pecuarias como el bovino, a nivel mundial la IA en porcinos con semen congelado, sólo se utiliza con fines experimentales, para intercambio de material genético entre países o conservación de germoplasma de altísimo mérito genético, debido a la disminución en las dosis procesadas, la fertilidad y el tamaño de la camada ${ }^{6,7,20}$.

Algunos investigadores indican que durante el congelamiento y descongelamien-to, los espermatozoides del porcino presentan cambios en la concentración y distribución de transportadores de glucosa, como el GLUT-3, en su membrana celular ${ }^{1}$. Su disminución podría explicar la corta viabilidad, alrededor de cuatro horas, de los espermatozoides descongelados del cerdo. Además la membrana plasmática del espermatozoide porcino tiene escasa resistencia al proceso de congelación-descongelación debido a su mayor concentración de fosfolípidos insaturados ${ }^{2}$.
Las inseminaciones utilizando espermatozoides congelados/descongelados resultan en bajas tasas de preñez y escaso tamaño de las camadas, comparadas con las obtenidas con semen fresco o refrigerado ${ }^{3}$.

Aún cuando la utilización de la inseminación intrauterina junto con la inseminación a tiempo fijo han permitido obtener mejores resultados ${ }^{8}$, la incorporación de esta biotecnología para el desarrollo productivocomercial se encuentra claramente limitada. La utilización de semen criopreservado no sólo tendría efectos a nivel productivo, como en la introducción de nueva genética, sino también a nivel sanitario ${ }^{6}$. De allí surge la importancia de posicionar no sólo el uso de la IA en porcinos en nuestro país sino también a la criopreservación de semen, como una tecnología de uso corriente en esta especie.

El objetivo de este trabajo fue comparar dos métodos de congelamiento para semen porcino, evaluando los parámetros de calidad seminal al momento del descongelado.

\section{MATERIAL Y MÉTODOS}

Obtención de las muestras de semen. Las mismas se recolectaron por la técnica de mano enguantada de 4 porcinos jóvenes mestizos (Yorkshire x Pietrain), de probada fertilidad. Los animales utilizados pertenecían a la Facultad de Ciencias Veterinarias de la Universidad de Buenos Aires, presentaban un estado corporal y sanitario óptimo y fueron mantenidos bajo condiciones uniformes de manejo y alimentación durante el tiempo que duró el estudio. Fueron congelados todos los eyaculados que presentaron un mínimo de $70 \%$ de espermatozoides móviles y un $80 \%$ de espermatozoides con morfología normal.

Congelamiento de las muestras de semen. Ellas fueron criopreservadas en pajuelas siguiendo un protocolo convencional ${ }^{13}$, realizando (grupo tratamiento) o no (grupo control) una modificación en el paso final de congelamiento posterior al envasado en las pajuelas. En forma resumida, las muestras a congelar se diluyeron a:a en medio Beltsville Thawing Solution (BTS) antes de pasar por un período de enfriamiento en dos etapas: primero a temperatura ambiente hasta $\operatorname{los} 25^{\circ} \mathrm{C}$ y luego, en heladera hasta $\operatorname{los} 15^{\circ} \mathrm{C}$. El período de estabilización se llevó a cabo a esta temperatura durante 2 horas.

Posteriormente, las muestras se centrifugaron a $300 \mathrm{G}$ por $3 \mathrm{~min}$, descartando el plasma seminal. El precipitado se re-suspendió en diluyente de enfriamiento (lactosa, yema de huevo, penicilina y estreptomicina) previamente a la realización de una curva de enfria- 
miento desde los $15^{\circ} \mathrm{C}$ hasta los $5^{\circ} \mathrm{C}$ en 1,5 horas (modificando la velocidad de enfriamiento a $\operatorname{los} 10^{\circ} \mathrm{C}$ y a $\operatorname{los} 8^{\circ} \mathrm{C}$ ). Una vez alcanzados $\operatorname{los} 5^{\circ} \mathrm{C}$ se realizó una segunda dilución con diluyente de congelamiento (lactosa, yema de huevo, Equex y glicerol), previo al envasado de las pajuelas. Luego del envasado, las pajuelas se mantuvieron $4 \mathrm{~min}$ a $-20^{\circ} \mathrm{C}$.

Finalmente, en el grupo "control", las pajuelas se congelaron sobre vapores de nitrógeno $(3 \mathrm{~min})$ y luego directamente en nitrógeno líquido, donde se almacenaron hasta su utilización. En el grupo "tratamiento" las pajuelas se congelaron colocándolas durante $1 \mathrm{~min}$ sobre una barra de hielo seco (para congelar el sellador) y luego, 10 min entre dos barras de hielo seco ${ }^{21}$. Las pajuelas de este grupo también se almacenaron en nitrógeno líquido hasta su utilización.

Determinación de la concentración espermática. La concentración espermática se determinó por hematocitometría en una cámara de Neubauer de $0,1 \mathrm{~mm}$ de profundidad.

Evaluación de la motilidad espermática. La evaluación de la motilidad del eyaculado y del semen descongelado se realizó por microscopía óptica según la rutina para inseminación artificial. En cada experiencia se evaluó el porcentaje de espermatozoides móviles a $37^{\circ} \mathrm{C}$, después de $10 \mathrm{~min}$ del atemperado, utilizando microscopia óptica en platina termostatizada.

Evaluación de la vitalidad espermática. Se ejecutó a través de dos métodos diferentes: la técnica de eosina/nigrosina ${ }^{14}$ y la combinación del colorante supravital azul tripán con el contraste interferencial diferencial ${ }^{5,11}$. Para determinar el porcentaje de espermatozoides vivos por la técnica de eosina/nigrosina se prepararon extendidos sobre portaobjetos, mezclando una alícuota de semen o suspensión espermática con el mismo volumen de eosina ( $1 \%$ en citrato de sodio al $1,92 \%)$ y de nigrosina $(10 \%$ en citrato de sodio al $1,92 \%)$ y se contaron 100 células por muestra.

Los espermatozoides muertos o moribundos presentaron un color rosado de diferente intensidad, dado que la membrana plasmática de los espermatozoides es impermeable a la eosina y sólo son coloreados los espermatozoides con serias alteraciones en la permeabilidad de sus membranas.

Para determinar el porcentaje de espermatozoides vivos por la técnica combinada de azul tripán y microscopía óptica con contraste interferencial-diferencial (DIC), a cada alícuota de suspensión espermática se le agregó igual volumen de azul tripán $(0,25 \%$ en medio TBM-Turkey Baster Method) y se incubó durante 15 min. Luego se centrifugó a $600 \mathrm{G}$ durante 5 min para remover el exceso de colorante y se fijó con 5\% de formaldehido en phosphate buffered saline (PBS) a pH 7,4.

Evaluación de la criocapacitación espermática. El porcentaje de espermatozoides criocapacitados se evaluó por la técnica fluorescente de cloro-tetraciclina (CTC ${ }^{4}$. Dicha técnica se basa en la capacidad de este antibiótico de quelar el calcio, indicando mediante fluorescencia, la localización de este ión unido a proteínas y glicoproteínas de la membrana plasmática ${ }^{17}$.

La solución de CTC fue preparada diariamente agregando $500 \mu \mathrm{M}$ de CTC al buffer que contenía $\mathrm{NaCl}$ $130 \mathrm{mM}$, cisteína $5 \mathrm{mM}$ y Tris $20 \mathrm{mM}, \mathrm{pH}=7,8$. La solución se mantuvo protegida de la luz hasta su utilización. A $100 \mu \mathrm{l}$ de la suspensión espermática se le agregaron $100 \mu \mathrm{l}$ de la solución de CTC. Finalmente se agregó glutaraldehído 12,5\% (v/v) en TRIS-HCl $1 \mathrm{mM}$, a una concentración final de $0,1 \%$ para fijación. Para evaluar la fluorescencia se utilizó un microscopio de epifluorescencia Carl Zeiss utilizando 410 ๆm de excitación de epifluorescencia, con una magnificación de 400x.

Esta técnica detecta cambios en la membrana plasmática del espermatozoide determinando tres patrones de fluorescencia: fluorescente $(F)$, espermatozoides intactos no capacitados que fluorescen en toda su superficie; capacitado (B), espermatozoides intactos y capacitados que perdieron la fluorescencia en la región postacrosomal; y reaccionados $(R A)$, espermatozoides con acrosomas reaccionados que perdieron la fluorescencia en las regiones acrosomal y post-acrosomal, expresándola sólo en la pieza intermedia y la cola.

Análisis estadístico: los diferentes parámetros evaluados en las muestras de espermatozoides como el porcentaje de espermatozoides vivos, porcentaje de espermatozoides con motilidad progresiva y porcentaje de espermatozoides criocapacitados, fueron expresados como porcentajes promedio \pm error estándar de la media (SEM). Los resultados de los diferentes experimentos fueron analizados por el test $t$ de student. En todos los casos, un valor de $\mathrm{p}<0,05$ fue considerado estadísticamente significativo.

\section{RESULTADOS}

El análisis estadístico de los resultados de las experiencias realizadas demostró que el método de congelamiento propuesto permite obtener, al descongelado, muestras de una mayor calidad que las obtenidas al descongelar muestras que hayan sido congeladas con el método tradicional.

Si bien la motilidad de los espermatozoides porcinos post-descongelado es muy baja (por eso uno de los objetivos de esta línea de investigación fue mejorar los métodos y los medios usados durante la criopreservación en esta especie), las muestras del grupo tratamiento evidenciaron una motilidad significativamente más alta que la del grupo control (Figura 1).

La criopreservación de las muestras con el método propuesto, prácticamente duplicó la vitalidad de los espermatozoides post descongelado. Tanto la vitalidad evaluada por la técnica supravital de eosina/nigrosina (Figura 2) como la vitalidad evaluada por azul tripán- 


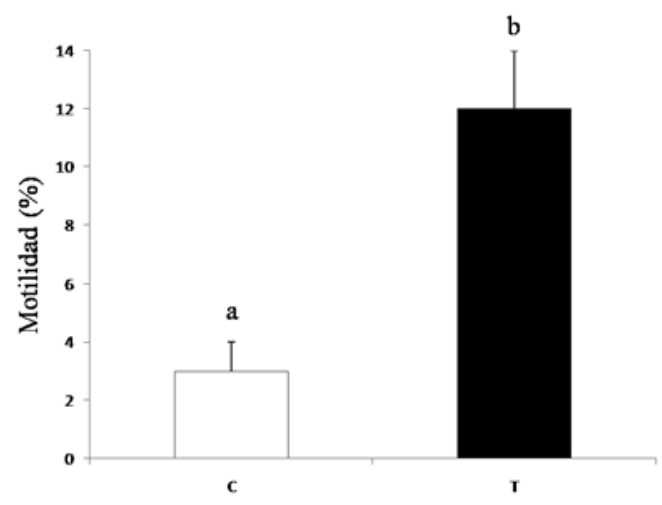

Figura 1. Efecto del método de congelamiento sobre la motilidad de espermatozoides porcinos.

C: grupo control; T: grupo tratamiento. Diferentes letras indican diferencias estadísticamente significativas, $\mathrm{p}<0,05(\mathrm{n}=6)$.

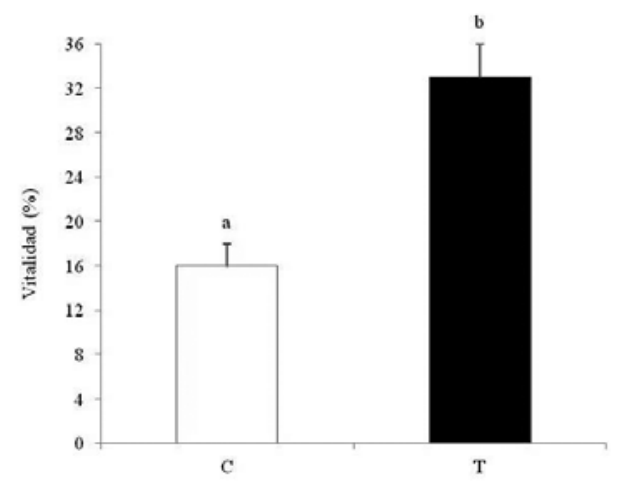

Figura 2. Efecto del método de congelamiento sobre la vitalidad de espermatozoides porcinos evaluada por la técnica de eosina/nigrosina.

C: grupo control; T: grupo tratamiento. Diferentes letras indican diferencias estadísticamente significativas, $\mathrm{p}<0,05(\mathrm{n}=6)$.

DIC, fue significativamente mayor en las muestras congeladas con el método propuesto que en grupo control (Figura 3).

Otro parámetro fuertemente afectado por el método de congelamiento fue la criocapacitación. Las muestras criopreservadas con el método propuesto (grupo tratamiento) evidenciaron una criocapacitación significativamente menor que las del grupo control (Figura 4).

\section{DISCUSIÓN}

Las metas de producción para el sector porcino presentadas en el Plan Estratégico Agroalimentario y Agroindustrial ${ }^{19}$ prevén un incremento en la productividad superior al $150 \%$. Esta mejoría en el sistema productivo, para ser eficiente, debe incorporar nuevas tecnologías y una adecuada y profesional gestión de recursos productivos que incluyen a las tecnologías reproductivas.

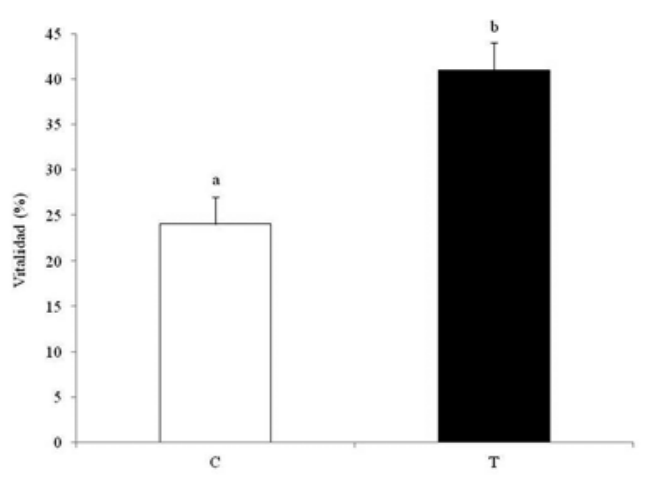

Figura 3. Efecto del método de congelamiento sobre la vitalidad de espermatozoides porcinos evaluada por la técnica de azul tripán-DIC.

C: grupo control; T: grupo tratamiento. Diferentes letras indican diferencias estadísticamente significativas, $\mathrm{p}<0,05(\mathrm{n}=6)$.

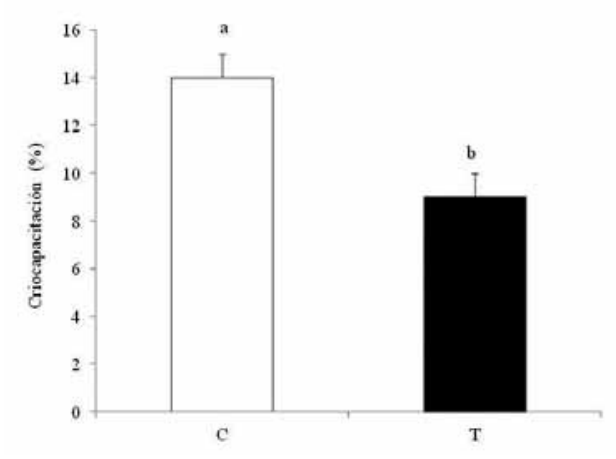

Figura 4. Efecto del método de congelamiento sobre la criocapacitación de espermatozoides porcinos. C: grupo control; T: grupo tratamiento. Diferentes letras indican diferencias estadísticamente significativas, $\mathrm{p}<0,05(\mathrm{n}=6)$.

Sin embargo, la aplicación de dichas innovaciones presenta dos problemas importantes: por un lado, si bien, en nuestro país, los niveles de producción se han incrementado en un $160 \%$ en la última década ${ }^{18}$, el $96 \%$ de los productores tienen menos de 50 madres 19 y en su mayoría producen en sistemas con baja inversión y poco tecnificados. Por otro lado, la producción de semen criopreservado porcino, para lograr características aceptables de calidad, requiere de la utilización de freezers programables para el congelamiento y otros medios de envasado especiales ${ }^{22}$ que no siempre se encuentran disponibles en nuestro medio.

En nuestro trabajo, hemos probado dos métodos de congelamiento para espermatozoides porcinos, que requieren tecnología habitualmente disponible en cualquier laboratorio de reproducción de baja complejidad. Las muestras de semen porcino criopreservado presentan alteraciones estructurales y funcionales con baja movilidad y una vitalidad de alrededor del $50 \%{ }^{23}$ debido en parte a que las membranas del espermatozoide 
porcino son muy sensibles al daño peroxidativo producido por el congelamiento debido al elevado número de ácidos grasos insaturados presentes en las mismas ${ }^{2}$.

De acuerdo a nuestros resultados, con el congelamiento profundo utilizando hielo seco se obtienen muestras con un adecuado nivel de integridad de membrana plasmática y acrosomal y bajo nivel de criocapacitación, pero con baja movilidad, posiblemente debido a la pérdida de enzimas vinculadas con las vías metabólicas productoras de ATP. Por tal motivo, las muestras criopreservadas obtenidas según el protocolo propuesto se podrían utilizar para ensayos de laboratorio (como protocolos de FIV o ICSI) o para fines productivos usando medios alternativos de IA como inseminación post cervical o intrauterina profunda, ya que estas técnicas utilizan un bajo número de espermatozoides ${ }^{22}$.

En conclusión, nuestros resultados demuestran que utilizando un método simple de congelamiento pueden obtenerse, al descongelado, muestras de una mayor calidad que las obtenidas al descongelar muestras que hayan sido congeladas con el método tradicional sobre vapores de nitrógeno.

\section{REFERENCIAS}

1. Bonet $\mathbf{S}$ et al. 2009. Biotecnología de la reproducción porcina: estado actual y futuro de las técnicas de análisis seminal. En: Biotecnología de la reproducción porcina, Ed. Universidad de Girona, España, p. 18-23.

2. Cerolini S, Maldjian A, Surai P, Noble R. 2000. Viability, susceptibility to peroxidation and fatty acid composition of boar semen during liquid storage. Anim Reprod Sci 58: 99-111.

3. Eriksson BM, Petersson H, Rodriguez MH. 2002. Field fertility with exported boar semen frozen in the new flatpack container. Theriogenology 58: 1065-1079.

4. Fraser LR, Abeydeera LR, Niwa K. 1995. Ca(2+)-regulating mechanisms that modulate bull sperm capacitation and acrosomal exocytosis as determined by chlortetracycline analysis. Mol Reprod Dev 40: 233-241.

5. Garde JJ, Ortiz N, García A, Gallego L. 1997. Use of a triple-stain technique to detect viability and acrosome reaction in deer spermatozoa. Arch Androl 39: 1-9.

6. Gerrits RJ et al. 2005. Perspectives for artificial insemination and genomics to improve global swine populations. Theriogenology 63: 283-299.

7. Iritani A. 1980. Problems of freezing spermatozoa of different species. Anales 9th Int Congr on Anim Reprod \& Artif Insem, Madrid, Vol. 1, p. 115-132.

8. Kaeoket $\mathbf{K}$, Chanapiwat $\mathbf{P}$, Wongtawan $\mathbf{T}$, Kunavongkrit A. 2010. Successful intrauterine insemination with frozen boar semen: effect of dose, volume and fixed-time AI on fertility. Thai Journ Agricult Sci 43: 31-37.
9. Khalifa $\mathbf{T}$ et al. 2014. Highlights on artificial insemination technology in the pigs. Mac Vet Rev 37: 5-34.

10. Ministerio de Hacienda y Finanzas Públicas. Secretaría de Política Económica y Planificación del Desarrollo (República Argentina). 2016. Informes de cadenas de valor Cárnica-Porcina. Año 1, № 9, p. 36.

11. O'Flaherty C, Beorlegui N, Beconi M. 1999. Reactive oxygen species requirements for bovine sperm capacitation and acrosome reaction. Theriogenology 52: 289-301.

12. OCDE-FAO. 2013. Perspectivas agrícolas 2013-2022, Texcoco, México. Universidad Autónoma Chapingo, http:// dx.doi.org/10.1787/agr_outlook-2013-es.

13. Peña FJ, Johannisson A, Wallgren M, Rodriguez MH. 2003. Antioxidant supplementation in vitro improves boar sperm motility and mitochondrial membrane potential after cryopreservation of different fractions of the ejaculate. Anim Reprod Sci 78: 85-98.

14. Pintado B, Fuente J, Roldan ER. 2000. Permeability of boar and bull spermatozoa to the nucleic acid stains propidium iodide or Hoechst 33258, or to eosin: accuracy on the assessment of cell viability. J Reprod Fertil 118: 145152.

15. Pordomingo A, Schang M, Brumori J. 2013. Plan Argentina Innovadora 2020. Documento ref. http://www.argentinainnovadora2020.mincyt.gob.ar/?wpfb_dl=52.

16. Romero C, Alba C, Martinez PC, Pascual MA. 2004. Situação atual de novas tecnologías na reprodução de suínos. SuínCia 2: 28.

17. Saling PA, Storey BT. 1979. Mouse gamete interactions during fertilization in vitro: chlortetracycline as a fluorescent probe for the mouse sperm acrosome reaction. $J$ Cell Biol 83: 544-555.

18. SENASA, MAGyP. 2014. Anuario 2014. www.produccion-animal.com.ar/produccion_porcina/00-produccion_ porcina_general/243-Anuario_2014.pdf

19. SENASA. 2016. Porcinos. Año 2016-2017. http://www. senasa.gov.ar/cadena-animal/porcinos/informacion/informes-y-estadisticas.

20. Williams S. 2005. Inseminación artificial en porcinos. En: Actualización de temas en Reproducción Animal (Bosch, R.), 2da. Ed., Univ.Nac.Río Cuarto, p. 397-417.

21. Wu TW et al. 2013. The combinatorial effect of different Equex STM paste concentrations, cryoprotectans and the straw-freezing methods on the post-thaw boar semen quality. Reprod Domest Anim 48: 53-58.

22. Yeste M, Rodríguez JE, Bonet S. 2017. Artificial insemination with frozen-thawed boar sperm. Mol Reprod Dev 84: 802-813.

23. Zeng WX, Shimada M, Isobe N, Terada T. 2001. Survival of boar spermatozoa frozen in diluents of varying osmolality. Theriogenology 56: 447-458. 